\title{
LOCAL THERMAL NON-EQUILIBRIUM MODELLING OF CONVECTIVE HEAT TRANSFER IN HIGH POROSITY METAL FOAMS
}

\author{
UBADE KEMERLI \& KAMIL KAHVECI \\ Department of Mechanical Engineering, Trakya University, Turkey
}

\begin{abstract}
In this paper, forced convective heat transfer in a rectangular channel filled with aluminium metal foam and exposed to a constant heat flux is examined numerically with the thermal non-equilibrium assumption. A constant heat flux boundary condition is applied from the upper side of the channel. A numerical model is first validated with the available experimental results. Next, the effects of different configurations of metal foams with different porosities and different PPI values on fluid flow and heat transfer are examined. Results are given by average Nusselt number and pressure drop factor for different Reynolds numbers. A performance factor is also defined and the effect of different configurations on performance factor is comparatively examined. The results show that the heat transfer rate and pressure drop significantly depending upon Reynolds number, configuration and porosity.
\end{abstract}

Keywords: metal foams, convection, heat transfer, modelling, local thermal non-equilibrium, pressure drop.

\section{INTRODUCTION}

In the last two decades, metal foams have been an important area of engineering research since they can be used in various engineering applications such as filters, catalyst supporters, energy absorbers, flame arresters and also for structural applications in buildings and vehicles. Researchers and engineers who study on heat exchangers and heat sinks also became interested in metal foams, due to their high heat transfer potential. The notable feature of metal foams is defined as the existence of many voids within the material by Han et al. [1]. There are two types of metal foams: open-cell metal foams and closedcell metal foams. While closed cell metal foams consist of individual enclosures [1], opencell metal foams are consisted of interconnected cells so the fluid flow can pass through it. While the louvered fins today are the known to be the most efficient and effective surface geometry in heat transfer point of view [2], metal foams present better heat transfer potential due to their high surface area to volume ratio and by preventing the growth of thermal boundary layers due to its complex structure and more fluid mixing. These features are demanded in heat transfer applications.

Since metal foams are interest of research, there are many studies in the literature about the thermal use of metal foams including many helpful review studies [3]-[7]. All of the studies related about the thermal use of metal foams show that using metal foams increase heat transfer. However, this increase comes with higher pressure drop. At this point, some of the studies claim that the metal foams can increase the heat transfer performance as well [8]. Yet some other studies claim the opposite [9], [10]. For instance, Ribeiro et al. [11] studied on the use of metal foams in condensers and found that the overall thermal conductance of the metal foam condensers was lower than that of plain fin condensers. Muley et al. [12] also studied on metal foam heat exchangers and they suggested new designs for reducing the excessive pressure drop. Hence, the idea of reducing pressure drop, while not sacrificing the heat transfer too much drives the researchers to find new designs. 
In some studies, instead of putting the metal foam directly in a channel, they were used in different configurations (e.g. partially filling the channel or corrugated filling). Sener et al. [13] experimentally investigated the forced convection in partial metal foam filled channels. They used triangular, convex and concave configurations inside the channel. Lin et al. [14] numerically investigated the different configurations inside of a heat exchanger for graphite foams. They achieved high thermal performance and low pressure drop by the wavy corrugated configuration. Also, there are some other studies which used graded pore size of metal foams [15], [16].

Since metal foams have complex geometric characteristics. It is very difficult to model them for numerical analyses. Although there are some studies in the literature which modelled metal foams geometrically [17], [18], they just modelled the small part of the metal foam. Volume averaging model was used for metal foams which was actually designed for porous media. In this model, there are two approaches; first one supposes that the solid and fluid part of the medium are in thermal equilibrium and uses effective thermal conductivity for the combined effect of fluid and solid zones. While latter method is based on the idea that the fluid and solid part of the medium cannot be assumed in thermal equilibrium. Hence, solid zones and fluid zones must be solved separately and a heat transfer coefficient between the solid and fluid zones must be defined and applied. The simplified energy equations for both models are as follows:

- LTE condition:

$$
\nabla . v\left(\rho C_{p} T\right)_{f}=\nabla k_{e f f} \nabla T .
$$

- Solid zone for LTNE condition:

$$
\nabla k_{s e} \nabla T_{s}=h_{s f} a_{s f}\left(T_{s}-T_{f}\right)
$$

- Fluid zone for LTNE condition:

$$
\nabla \cdot v\left(\rho C_{p}\right)_{f}=\nabla\left(k_{f e}+k_{d}\right) \nabla T_{f}+k_{f s} a_{f s}\left(T_{s}-T_{f}\right) .
$$

The difference can be seen in the equations of these models. In eqns (2) and (3), three extra terms can be seen which the $\mathrm{h}_{\mathrm{fs}}$ is the heat transfer coefficient between the solid and fluid and $a_{f s}$ is the interface area between fluid and solid state and $k_{d}$ is the thermal dispersion conductivity. Thermal dispersion is an important factor affecting heat transfer in porous media [19].

The main drawback here is to define $h_{f s}$ and $k_{d}$ values. Since $h_{f s}$ and $k_{d}$ vary inside of the metal foam with the velocity. Assuming constant $h_{f s}$ and $k_{d}$ values will not result an accurate solution. Calmidi and Mahajan [20] studied on this problem. They prepared an experimental setup and also they modelled this experimental setup numerically in 2D. For $\mathrm{k}_{\mathrm{d}}$ and $\mathrm{h}_{\mathrm{fs}}$ values, they proposed following correlations:

$$
\begin{gathered}
\frac{k_{\text {st }}}{k_{e}}=C_{D}\left(R e_{K} P \tau_{e}\right) \frac{u}{w_{0}} \\
N u_{s f}=\frac{h_{s f} b_{f}}{k_{f}}=C_{T} R e^{0.5} P r^{0.37},
\end{gathered}
$$

Here, there are two new parameters; $C_{D}$ and $C_{T}$. They obtained these two values by benchmarking the numerical results against the experimental data and suggested 0.52 for $C_{T}$ and 0.06 for $C_{D}$. 
In this study, same geometry will be modelled in 3D, then numerically solved and validated first with the experimental data in the literature. Then, different configurations will be investigated. It must be noted that the numerical model of Calmidi is limited to 2D, yet this model will be a 3D model, hence some differences are expected.

\section{MODEL}

\subsection{Samples and configurations}

In this study, there are four types of geometries. Configuration 1 is modelled for validation; other geometries are studied for comparison between each other. In Fig. 1, different configurations can be seen while in Table 1, eight different situations are given.

As it can be seen in Table 1, there are four different configurations and two different samples. Therefore, there are 8 situations which will be examined for different inlet velocities. Configuration 1 is designed identically to the geometry used in the study of Calmidi. Hence, this configuration is used for validation.

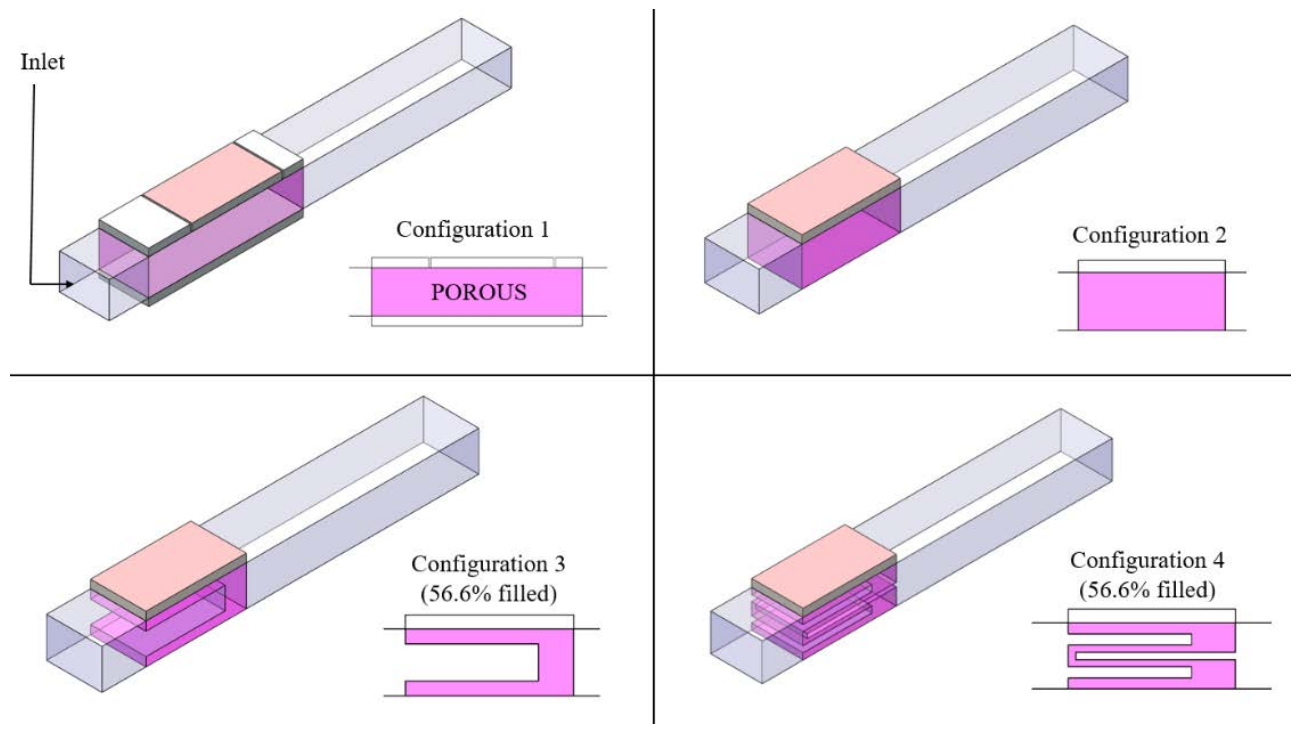

Figure 1: Different configurations of metal foams inside the channel.

Table 1: Samples and geometries.

\begin{tabular}{|l|c|c|}
\hline \multirow{2}{*}{ Configuration } & \multicolumn{2}{|c|}{ Different samples } \\
\cline { 2 - 3 } & Sample 1 & Sample 4 \\
\hline Configuration 1 & G1S1 & G1S4 \\
\hline Configuration 2 & G2S1 & G2S4 \\
\hline Configuration 3 & G3S1 & G3S4 \\
\hline Configuration 4 & G4S1 & G4S4 \\
\hline
\end{tabular}


Table 2: Characteristics of the metal foam samples. (Source: Calmidi and Mahajan [20].)

\begin{tabular}{|c|c|c|c|c|c|c|c|c|}
\hline$\#$ & Porosity & PPI & $d_{f(\mathrm{~m})}$ & $d_{p(\mathrm{~m})}$ & $f$ & $\mathrm{~K}$ & $k_{s e}$ & $k_{f e}$ \\
\hline 1 & 0.9726 & 5 & 0.0005 & 0.00402 & 0.097 & 2.7 & 2.48 & 0.0256 \\
\hline 4 & 0.9546 & 20 & 0.0003 & 0.00270 & 0.093 & 1.3 & 3.71 & 0.0250 \\
\hline
\end{tabular}

The two samples are selected from the experimental study of Calmidi. Some values of the samples can be seen in Table 2. The numbers of samples remained the same with the experimental study.

\subsection{Numerical model}

LTNE model is used in FLUENT for numerical solutions. For porous media, $\mathrm{k}_{\mathrm{d}}$ and $\mathrm{h}_{\mathrm{fs}}$ values are applied by using UDF codes utilizing the eqns (4) and (5). Flow is assumed laminar therefore, another UDF code is applied for obtaining laminar inlet velocity profile. A heat flux boundary condition is applied from the upper side of the channel. Applied heat flux is selected as $1500 \mathrm{~W} / \mathrm{m}^{2}$ for all situations. Grid sizes are selected in order to comply with the $2 \mathrm{D}$ numerical model in the literature.

\subsection{Evaluation of parameters}

The Reynolds number is defined based on permeability as follows

$$
R e=\frac{\rho u_{0} \Omega}{\mu} .
$$

The Nusselt number is defined as

$$
N u=\frac{\pi_{L}}{\kappa_{e}}
$$

While $h$ is defined as follows

$$
\bar{h}=q /\left(A \Delta T_{\text {awg }}\right)
$$

$\Delta T_{\text {avg }}$ is defined as the temperature difference between the base and inlet. $L$ is the length of the porous zone parallel to the flow direction and ke is the effective thermal conductivity which was defined as

$$
k_{e}=k_{f e}+k_{s e} \text {. }
$$

Friction factor is defined as follows

$$
f=\frac{2 D_{h}\left(\Delta P{ }_{h}\right)}{\rho u_{0}^{2}}
$$

A performance factor is also defined based on the literature [21] of cellular metals as follows

$$
\text { Performance factor }=N{ }^{N} / f^{1 / 3^{*}}
$$




\section{RESULTS}

The results first obtained for configuration 1 in order to validate the results. In Fig. 2, the numerical results and the experimental results in the literature can be seen. Simulation results were well matched with the experimental data. For higher Re numbers, numerical results start to underpredict the experimental results.

Temperature variations in the fluid zone can be seen in Figs 3-5. As it can be seen in Fig. 3, bottom of the porous zone remained cold. This shows that the bottom part of the metal foam is not used efficiently. While in configuration 3 and 4 , the temperature gradient is more prominent.
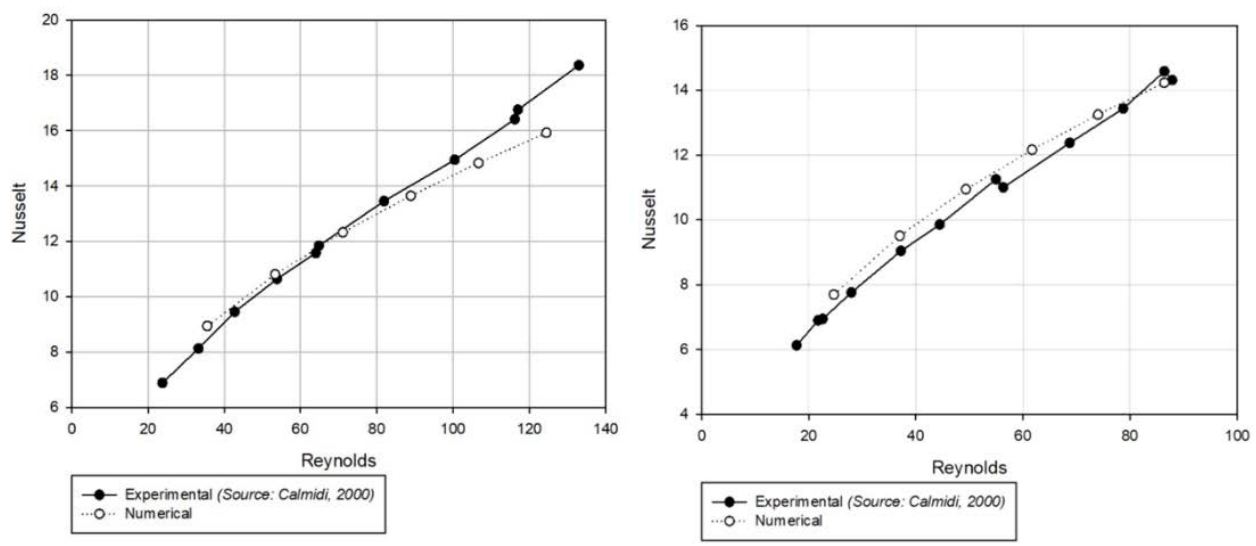

Figure 2: Validation for Sample 1 (left) and Sample 4.

[C]
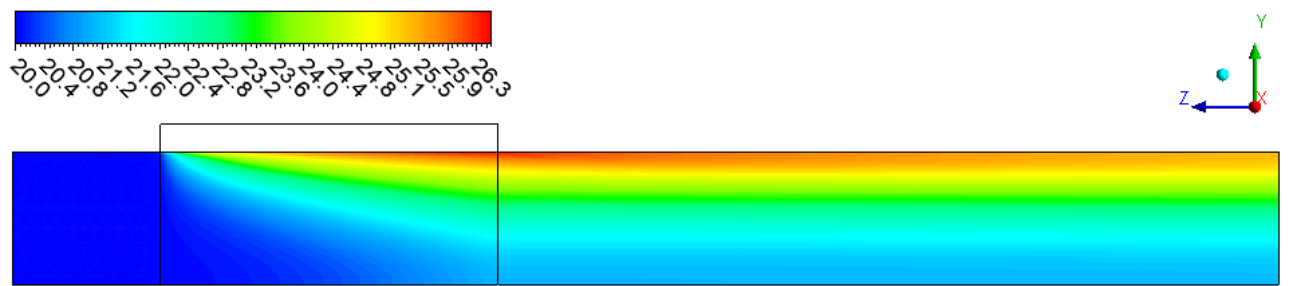

Figure 3: Temperature variation in fluid zone for G2S4 (inlet velocity: 1m/s).

[C]

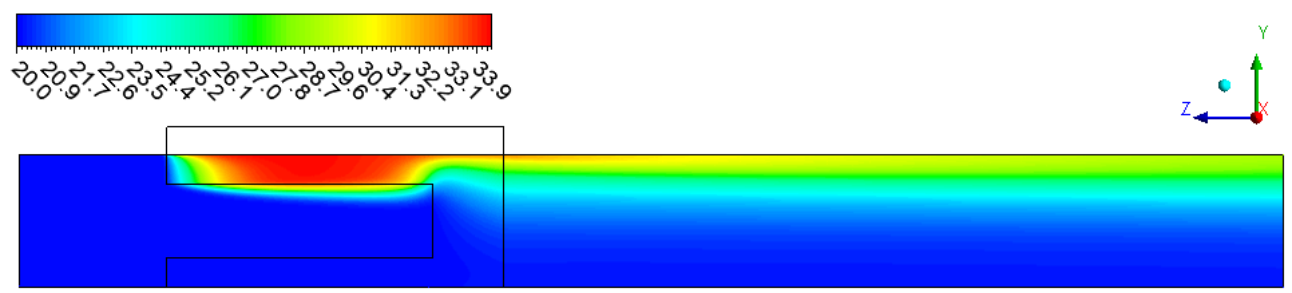

Figure 4: Temperature variation in fluid zone for G3S4 (inlet velocity: 1m/s). 
[C]
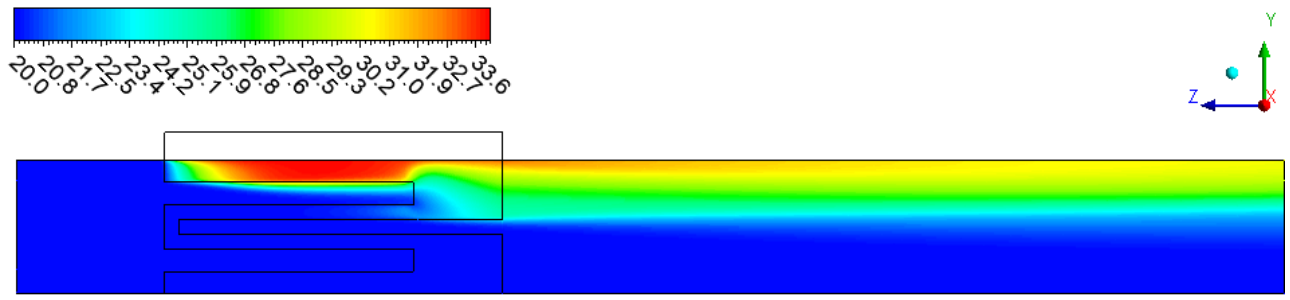

Figure 5: Temperature variation in fluid zone for G4S4 (inlet velocity: 1m/s).

In Fig. 6, Nusselt numbers for different configurations can be seen. As expected, Configuration 1 has the highest $\mathrm{Nu}$ value for both samples. Configuration 4 has a higher Nusselt number than Configuration 3 for both samples. Nusselt number for Configuration 2 is about 2 times higher than other configurations for both samples. While two samples are compared between each other, the Nusselt number is higher for Sample 1.

In Fig. 7, f values can be seen. For both samples, configuration 2 is the highest friction factor as it is expected. Configuration 4 has also higher friction factor than the one for Configuration 3. Yet, this difference is less prominent for Sample 4. Friction factor for Sample 4 is higher than the friction factor for Sample 1 which has higher porosity and lower PPI value.

In Fig. 8, performance factor values can be seen. As it can be observed, for both samples, configuration 2 has the highest performance factor. However, it must be noted that configuration 3 and 4 have less metal foam material. Latter two configurations have $56.6 \%$ material used compared to Configuration 1 while their performance factor is about $80 \%$ of configuration 1 . This means that the material is used more efficiently for configuration 3 and 4 . While comparing the configuration 3 and 4 between each other. Configuration 4 seems to be slightly more advantageous than Configuration 3 especially for Sample 4 . When comparing both samples, Sample 1 seems to have higher PF than Sample 4.
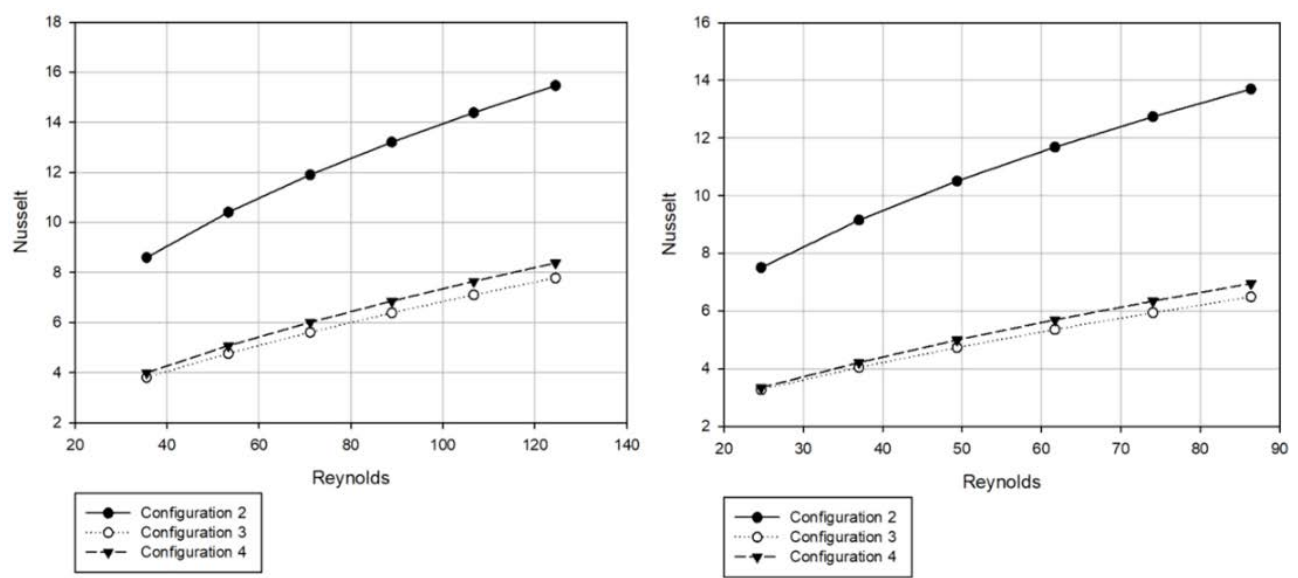

Figure 6: Nusselt numbers for different configurations for Sample 1 (left) and Sample 4. 

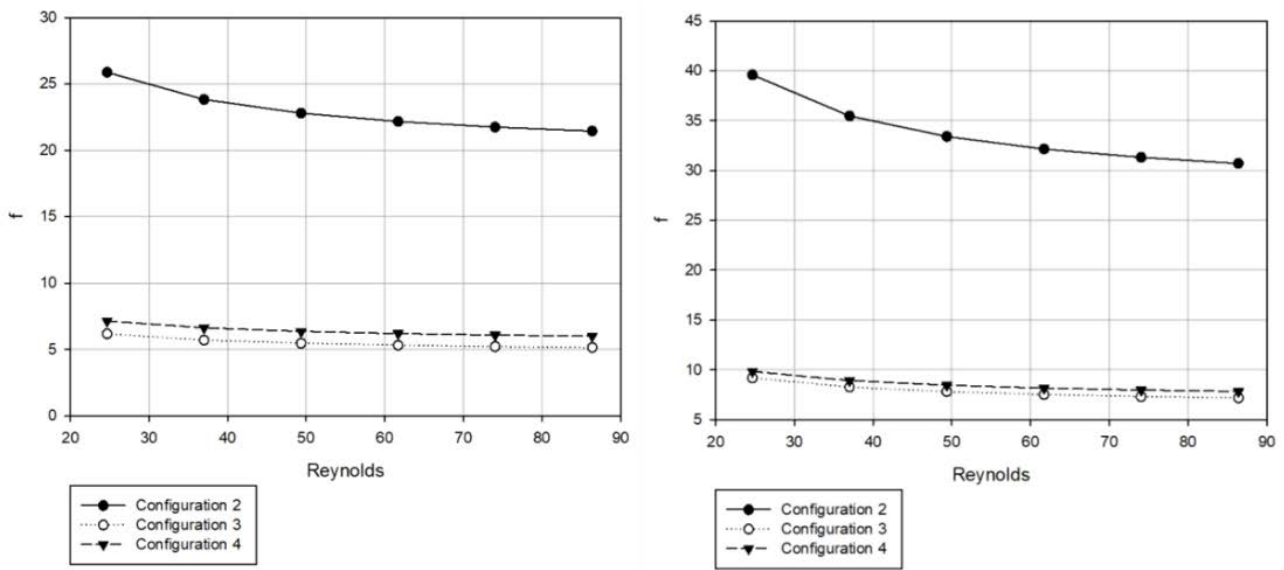

Figure 7: Friction factor for different configurations for Sample 1 (left) and Sample 4.
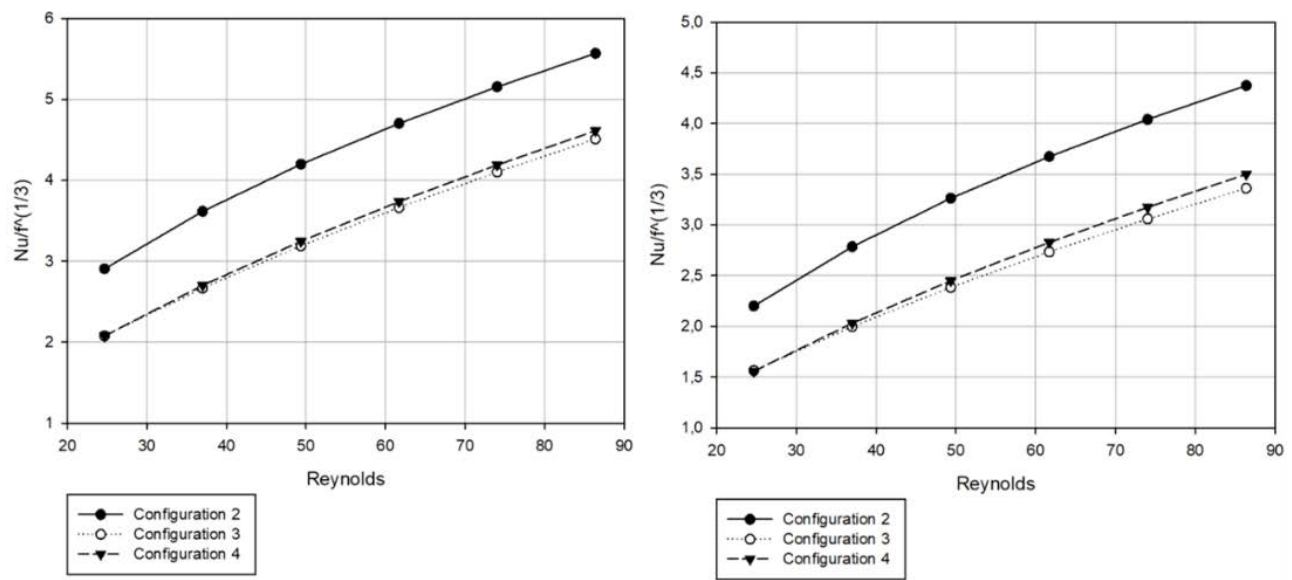

Figure 8: Performance factor for different configurations for Sample 1 (left) and Sample 4.

\section{CONCLUSION}

Two different samples with different porosity and PPI values are examined for three different configurations. From the results, it can be concluded that:

- The heat transfer rate and pressure drop significantly depend on Reynolds number, configuration and porosity.

- Using the outlying zones of the material efficiently is an important point, since heat cannot be transferred properly through porous solid.

- Configuration 3 and 4 use the material in a more efficient way comparing to Configuration 1.

- Results show that there are more efficient designs available for metal foams to achieve higher efficiency. 


\section{REFERENCES}

[1] Han, X.-H., Wang, Q., Park, Y.-G., T'Joen, C., Sommers, A. \& Jacobi, A., A review of metal foam and metal matrix composites for heat exchangers and heat sinks. Heat Transfer Engineering, 33(12), pp. 991-1009, 2012.

[2] Kim, S., Paek, J. \& Kang, B., Flow and heat transfer correlations for porous fin in a plate-fin heat exchanger. Journal of Heat Transfer, 122(3), pp. 572-578, 2000.

[3] de Schampheleire, S., de Jaeger, P., de Kerpel, K., Ameel, B., Huisseune, H. \& de Paepe, M., How to study thermal applications of open-cell metal foam: Experiments and computational fluid dynamics. Materials, 9(2), p. 94, 2016.

[4] Banhart, J., Metallic foams: Challenges and opportunities. Eurofoam, 2000, pp. 1320, 2000.

[5] Ozmat, B., Leyda, B. \& Benson, B., Thermal applications of open-cell metal foams. Materials and Manufacturing Processes, 19(5), pp. 839-862, 2004.

[6] Ghosh, I., How good is open-cell metal foam as heat transfer surface? Journal of Heat Transfer, 131(10), p. 101004, 2009.

[7] Baloyo, J.M., Open-cell porous metals for thermal management applications: Fluid flow and heat transfer. Materials Science and Technology, pp. 1-12, 2016.

[8] Mahjoob, S. \& Vafai, K., A synthesis of fluid and thermal transport models for metal foam heat exchangers. International Journal of Heat and Mass Transfer, 51(15-16), pp. 3701-3711, 2008.

[9] Sertkaya, A.A., Altınısık, K. \& Dincer, K., Experimental investigation of thermal performance of aluminum finned heat exchangers and open-cell aluminum foam heat exchangers. Experimental Thermal and Fluid Science, 36, pp. 86-92, 2012.

[10] Mancin, S., Zilio, C., Rossetto, L. \& Cavallini, A., Heat transfer performance of aluminum foams. Journal of Heat Transfer, 133(6), 060904, 2011.

[11] Ribeiro, G.B., Barbosa, J.R. \& Prata, A.T., Performance of microchannel condensers with metal foams on the air-side: Application in small-scale refrigeration systems. Applied Thermal Engineering, 36, pp. 152-160, 2012.

[12] Muley, A., Kiser, C., Sundén, B. \& Shah, R.K., Foam heat exchangers: A technology assessment. Heat Transfer Engineering, 33(1), pp. 42-51, 2012.

[13] Sener, M., Yataganbaba, A. \& Kurtbas, İ., Forchheimer forced convection in a rectangular channel partially filled with aluminum foam. Experimental Thermal and Fluid Science, 75, pp. 162-172, 2016.

[14] Lin, W., Sundén, B. \& Yuan, J., A performance analysis of porous graphite foam heat exchangers in vehicles. Applied Thermal Engineering, 50(1), pp. 1201-1210, 2013.

[15] Zaragoza, G. \& Goodall, R., Metal foams with graded pore size for heat transfer applications. Advanced Engineering Materials, 15(3), pp. 123-128, 2013.

[16] Carpenter, K.P. \& da Silva, A.K., A combined hydro-thermal characterization of high-porosity metal foam test sections with discrete pore-size gradients. International Journal of Heat and Mass Transfer, 77, pp. 770-776, 2014.

[17] Al-Athel, K.S., A computational methodology for assessing the thermal behavior of metal foam heat sinks. Applied Thermal Engineering, 111, pp. 884-893, 2017.

[18] Zafari, M., Panjepour, M., Davazdah Emami, M. \& Meratian, M., Microtomographybased numerical simulation of fluid flow and heat transfer in open cell metal foams. Applied Thermal Engineering, 80, pp. 347-354, 2015.

[19] Hsu, C. \& Cheng, P., Thermal dispersion in a porous medium. International Journal of Heat and Mass Transfer, 33(8), pp. 1587-1597, 1990. 
[20] Calmidi, V. \& Mahajan, R., Forced convection in high porosity metal foams. Journal of Heat Transfer, 122(3), pp. 557-565, 2000.

[21] Tian, J. et al., The effects of topology upon fluid-flow and heat-transfer within cellular copper structures. International Journal of Heat and Mass Transfer, 47(1416), pp. 3171-3186, 2004. 